\title{
Circulating concentrations of asymmetrical dimethyl-L-arginine are increased in women with previous gestational diabetes
}

\author{
F. Mittermayer ${ }^{1}$, B. X. Mayer ${ }^{1}$, A. Meyer ${ }^{1}$, C. Winzer ${ }^{2}$, G. Pacini ${ }^{4}$, O. F. Wagner ${ }^{3}$, M. Wolzt ${ }^{1}$, \\ A. Kautzky-Willer ${ }^{2}$ \\ ${ }^{1}$ Department of Clinical Pharmacology, ${ }^{2}$ Department of Internal Medicine III, ${ }^{3}$ Clinical Institute for Medical \\ and Chemical Laboratory Diagnostics, University of Vienna, Vienna, Austria \\ ${ }^{4}$ Institute of Systems Science and Biomedical Engineering (LADSEB-CNR), Padova, Italy
}

\section{Abstract}

Aims/hypothesis. The concentration of asymmetrical dimethyl-L-arginine (ADMA), an endogenous inhibitor of the nitric oxide synthase, is increased in patients at risk or with cardiovascular disease. We have investigated ADMA concentrations in women with a history of gestational diabetes (GDM), who could develop endothelial dysfunction and Type II (non-insulin-dependent) diabetes mellitus after delivery, and in healthy control subjects.

Methods. Previous GDM patients were grouped according to their BMI as obese $\left(\geq 25 \mathrm{~kg} / \mathrm{m}^{2}, n=46\right)$ or non-adipose $\left(<25 \mathrm{~kg} / \mathrm{m}^{2}, n=31\right)$. Serum samples were taken 14 to 16 weeks after delivery and after 1 year. The control group comprised 17 healthy women $\left(\mathrm{BMI}<25 \mathrm{~kg} / \mathrm{m}^{2}\right)$. ADMA concentrations were analysed by high performance liquid chromatography.

Results. ADMA concentrations were comparable between obese and non-adipose GDM patients $(0.58 \pm 0.02$ and $0.57 \pm 0.02 \mu \mathrm{mol} / 1$, respectively), and higher than in the control group $(0.47 \pm 0.03 \mu \mathrm{mol} / \mathrm{l}$; $p<0.006)$. Insulin resistance as estimated by the insulin sensitivity index was more frequent among the obese than the non-adipose GDM women $(p<0.05)$ and control subjects $(p<0.05$, both). No change in ADMA concentrations was found after 1 year in women with GDM. There was only a slight correlation between ADMA and BMI $(r=0.26, p<0.02)$, triglycerides $(r=0.29, p<0.004)$, or fasting plasma glucose $(r=0.21, p<0.05)$, and not with the insulin sensitivity index or other parameters. In a multiple regression analysis ADMA serum concentrations were only associated with triglycerides.

Conclusion/interpretation. Circulating ADMA concentrations are increased in normoglycaemic women with previous GDM. This increase is independent from other risk factors or surrogate markers for diabetes or cardiovascular events. [Diabetologia (2002) 45:1372-1378]

Keywords Gestational diabetes, asymmetrical dimetylarginine, nitric oxide, insulin resistance, cholesterol.
Nitric oxide is synthesized from the amino acid L-arginine by constitutive and inducible nitric oxide synthases [1] and plays an important role in the regulation

Received: 22 March 2002 / Revised: 10 May 2002

Published online: 16 August 2002

C) Springer-Verlag 2002

Corresponding author: Dr. M. Wolzt, Department of Clinical Pharmacology, Allgemeines Krankenhaus Wien, Währinger Gürtel 18-20, 1090 Vienna, Austria. E-mail: michael.wolzt@univie.ac.at Abbreviations: ADMA, Asymmetrical dimethyl-L-arginine; SDMA, symmetrical dimethyl-L-arginine; GDM, gestational diabetes mellitus. of vascular tone, thrombocyte activation, neurotransmission and host defence [2]. L-arginine can also be methylated by intracellular methyltransferases [3], but the exact regulation of this pathway is not known. The methylated L-arginine metabolite, asymmetrical dimethyl-L-arginine (ADMA), inhibits the cellular L-arginine uptake and nitric oxide synthase activity in endothelial cells competitively [4]. The allosteric symmetrical dimethyl-L-arginine (SDMA) is produced in equivalent amounts, but is not a structural antagonist in nitric oxide synthesis [5].

It has been speculated that accumulation of endogenous L-arginine metabolites and low availability of L- 
arginine could reduce nitric oxide formation and influence vascular function. A decreased plasma L-arginine to ADMA ratio or increased ADMA concentrations has been described in hypercholesterolemia $[6,7]$, hypertension [8, 9], arterial occlusive disease [10], chronic renal failure [11], and preeclampsia [12]. It has been suggested that alteration of the L-arginine to ADMA ratio could affect the clinical course of arteriosclerosis, which has not been seen in patients with stable angina [13].

Endothelial dysfunction is typically found in patients prone to develop arteriosclerosis such as in Type I (insulin-dependent) and Type II (non-insulin-dependent) diabetes mellitus $[14,15]$ and in women with previous gestational diabetes (GDM) $[16,17]$ who are at risk of developing Type II diabetes. In Type II diabetes, an increase in ADMA has also been described [18], and insulin resistance was recently reported to be related to ADMA in non-diabetic normotensive subjects [19]. However, the serum concentrations of ADMA, which could be used to monitor early changes in the L-arginine-NO metabolism, have not been studied in GDM patients. We have quantified serum ADMA concentrations in otherwise healthy women with a history of GDM and in age-matched control subjects.

\section{Subjects and methods}

This cross sectional study was approved by the Ethics Committee of the University of Vienna and complies with the Declaration of Helsinki including current revisions and the GCP guidelines. Written informed consent was obtained from all subjects prior to enrolment.

Subjects. All women with GDM, diagnosed as part of their clinical tests during their 24th to 28th week of pregnancy at the Department of Internal Medicine III between August 1999 and June 2001, were invited to participate in the study. GDM was defined as two or more of the following criteria: fasting morning plasma glucose after an overnight fast and at least 3 days of unrestricted diet $(\geq 150 \mathrm{~g}$ carbohydrate per day) and unlimited physical activity of $95 \mathrm{mg} / \mathrm{dl}$ or more, 1 hour postload $75 \mathrm{~g}$ oral glucose of $180 \mathrm{mg} / \mathrm{dl}$ or more, and 2 hours postload glucose of $155 \mathrm{mg} / \mathrm{dl}$ or more. Inclusion criteria were signed consent, absence of a clinically relevant illness, normal findings in the medical history except for GDM and in the physical examination, and normal laboratory values including plasma homocysteine concentrations in a complete health examination. Subjects were excluded if any clinically relevant abnormality was found as part of the screening or in any of the laboratory tests including circulating anti-insulin antibodies and anti-islet cell antibodies or if they participated in a different clinical trial.

77 otherwise healthy women with a history of GDM and fasting glucose concentrations of less than $110 \mathrm{mg} / \mathrm{dl}$ were included in the study. Women with GDM were grouped according to BMI, which is associated with insulin resistance and diabetes mellitus [20, 21]. Patients were defined as overweight with a BMI of $25 \mathrm{~kg} / \mathrm{m}^{2}$ or more (obese, $n=46$ ), and normal with a BMI of less than $25 \mathrm{~kg} / \mathrm{m}^{2}$ (non adipose, $n=31$ ), respectively [22]. The control group comprised 17 age-matched heal- thy women with a BMI of less than $25 \mathrm{~kg} / \mathrm{m}^{2}, 13$ had one or more pregnancies and did not have a history of GDM and four women had no previous pregnancy. Blood samples were drawn at the same time after delivery.

No subject was on a special diet or reported intake of any medication, including 'over-the-counter' drugs, at the time of blood sampling or had a history or presence of disorders associated with increased ADMA concentrations such as renal insufficiency or arteriosclerosis. Blood pressure was measured by sphygmomanometry (mean of three measurements in resting sitting position).

Study protocol. Samples for measurement of serum ADMA, SDMA, plasma triglycerides, total and HDL and LDL cholesterol, and fasting glucose concentrations were taken between week 14 and 16 after delivery. Samples from 48 women with previous GDM (obese, $n=24$, non-adipose, $n=24$ ) were also available after a 1-year follow up.

A 75 g OGTT was carried out after overnight fasting. Venous plasma glucose concentrations were measured before and $1 \mathrm{~h}$ after the oral glucose load. Insulin resistance was evaluated by the insulin sensitivity index $\left(\mathrm{S}_{\mathrm{I}}\right)$ during an insulin-modified intravenous glucose tolerance test (FSIGT) [23]. An $S_{I}$ value of $3 \cdot 10^{-4} \mathrm{~min}^{-1} \cdot \mathrm{ml} \cdot \mu \mathrm{U}^{-1}$ was used to discriminate normal $\left(\mathrm{S}_{\mathrm{I}} \geq 3 \cdot 10^{-4} \mathrm{~min}^{-1} \cdot \mathrm{ml} \cdot \mu \mathrm{U}^{-1}\right)$ from impaired insulin sensitivity $\left(\mathrm{S}_{\mathrm{I}}<3 \cdot 10^{-4} \mathrm{~min}^{-1} \cdot \mathrm{ml} \cdot \mu \mathrm{U}^{-1}\right)$, respectively. This value was derived from a historical female control cohort $(n=22)$, where less than $5 \%$ of $\mathrm{S}_{\mathrm{I}}$ values were allowed to lie outside the normal range $(p<0.05)$. When the lower $2.5 \%$ quartile was taken as cut-off between normal and impaired $S_{I}$, the normal reference limit was $2.1 \cdot 10^{-4} \mathrm{~min}^{-1} \cdot \mathrm{ml} \cdot \mu \mathrm{U}^{-1}$. In our study, the conservative choice with an $\mathrm{S}_{\mathrm{I}}$ of $3 \cdot 10^{-4} \mathrm{~min}^{-1} \cdot \mathrm{ml} \cdot \mu \mathrm{U}^{-1}$ was defined as normal. Standard laboratory parameters were quantified according to certified routine methods at the Clinical Institute for Medical and Chemical Laboratory Diagnostics, AKH (Vienna, Austria).

Measurement of ADMA and SDMA. Venous blood from fresh venipuncture was centrifuged, separated, and stored at $-30^{\circ} \mathrm{C}$ until batch analysis. Serum ADMA and SDMA concentrations were quantified by high performance liquid chromatography (HPLC) with $o$-phtaldialdehyde (OPA) precolumn fluorescence derivatization using a modified method described previously [24]. $N^{\mathrm{G}}, N^{\mathrm{G}}$-dimethyl-L-arginine (ADMA), $N^{\mathrm{G}}, N^{\prime} \mathrm{G}$-dimethylL-arginine (SDMA) were obtained from Calbiochem (Darmstadt, Germany), OPA was obtained from Pierce (Rockford, Ill., USA), 2-mercaptoethanol and trichloracetic acid were obtained from Merck (Darmstadt, Germany). The HPLC system was a System Gold (solvent module 126, autosampler 528, Beckman-Coulter, Fullerton, Calif., USA) with 32 Karat software. ADMA and SDMA were extracted from serum by solid phase extraction. The column (Isolute, SCX $100 \mathrm{mg}, 1 \mathrm{ml}$, Mid Glamorgan, UK) was activated with methanol, conditioned with TCA $(2 \%)$ and loaded with sample. Subsequently the column was rinsed with TCA (2\%), phosphate buffer ( $\mathrm{pH} 8)$ and methanol. ADMA and SDMA were eluted in methanol containing triethylamine prepared fresh daily. The eluent was evaporated to dryness at $+45^{\circ} \mathrm{C}$ and 1 mbar. The dried extract was redissolved in distilled water. The $o$-phthaldialdehyde precolumn derivatization was done in the autosampler for $1 \mathrm{~min}$ at $+8^{\circ} \mathrm{C}$. ADMA and SDMA were separated using a multigradient program on a Synergi $4 \mu$ MAX-RP 80 A column $(150 \times 2.00 \mathrm{~mm}$, Phenomenex, Torrance, Calif., USA) with a Security Guard Max-RP guard column (4 mm 1 x $3.0 \mathrm{~mm}$ ID, Phenomenex, Torrance, Calif., USA) using a binary gradient. Solvent A was $15 \mathrm{mmol} / \mathrm{l}$ phosphate buffer ( $\mathrm{pH} 7.0)$, containing 5\% methanol and $5 \%$ tetrahydrofuran $(\mathrm{v} / \mathrm{v})$ and solvent $\mathrm{B}$ was $40 \%$ phos- 
phate buffer $(15 \mathrm{mmol} / \mathrm{l}), 45 \%$ methanol and $15 \%$ acetonitrile $(\mathrm{v} / \mathrm{v})$. The fluorescence detector, with excitation set at $330 \mathrm{~nm}$ and emission at $440 \mathrm{~nm}$ was used for detection. The elution times were approximately 53.3 and 54.2 min for ADMA and SD MA, respectively. A calibration curve constructed with standards in water was used because the internal standard DLhomoarginine eluted with SDMA in pilot experiments. The relation between concentration and peak area was linear in the range from $0.156 \mu \mathrm{mol} / 1$ to $5 \mu \mathrm{mol} / 1$ for both ADMA and SDMA (regression coefficient $>0.999$ ). The limit of quantification (LOQ) was $0.078 \mu \mathrm{mol} / \mathrm{l}$. The recovery rate was checked with standards in water and spiked plasma samples and was $81 \pm 8 \%$. The coefficient of variation of the autosampler was $3.0 \%$, and the intra-sample variation $5.7 \%$.

Statistical analysis. Outcome parameters were tested for normal distribution and log transformed if not normally distributed. Between and within group differences were analysed by a Student's unpaired or paired $t$ test. Correlations between outcome parameters were calculated for pooled data sets and subgroups using Pearson's correlation and a multiple regression analysis was applied. A $p$ value of less than 0.05 was considered statistically significant. Data are presented as means \pm SEM

\section{Results}

Baseline data are shown in Table 1. Obese women had higher blood pressure, triglycerides and fasting glucose concentrations and lower HDL cholesterol concentrations than non-adipose women with previous GDM $(p<0.05)$ and healthy control subjects $(p<0.05)$. Stimulated 1-h glucose concentrations were also higher in obese $(p<0.00002)$ and in non-adipose GDM women $(p<0.01)$ than in the control subjects. Creatinine concentrations were lower in obese $(p<0.0001)$

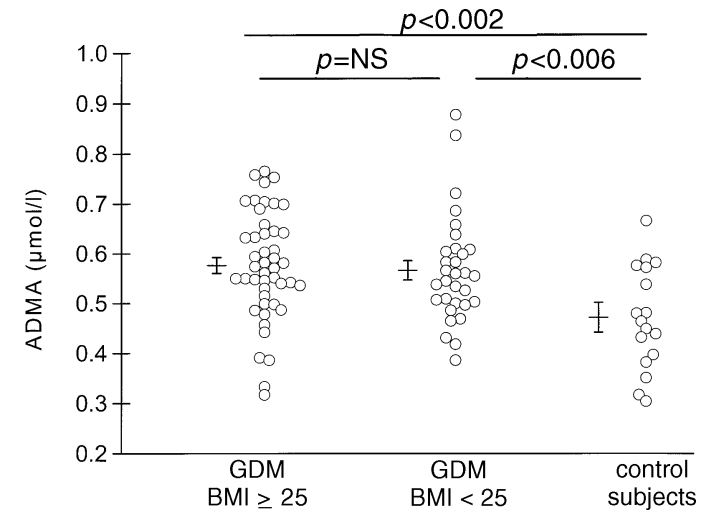

Fig. 1. Individual ADMA concentrations and means \pm SEM in women with a history of GDM and BMI $\geq 25 \mathrm{~kg} / \mathrm{m}^{2}$ or $<25 \mathrm{~kg} / \mathrm{m}^{2}$ and healthy control subjects. Statistical differences between groups are indicated, unpaired Student's $t$ test

and non-adipose GDM women $(p<0.01)$ compared to the healthy control subjects. Insulin resistance was more frequent among obese than non-adipose GDM women $(p<0.05)$ and control subjects $(p<0.05$, both).

ADMA serum concentrations were not different between obese and non-adipose women with previous GDM, but higher than in the control subjects $(p<0.006$, both groups; Fig. 1). SDMA was lower in obese than in non-adipose GDM patients $(p<0.04)$. The ADMA-to-SDMA ratio was higher in the obese compared to non-adipose women $(p<0.01)$ and control subjects $(p<0.002)$.

There was only a slight correlation between ADMA concentrations and BMI $(r=0.26, p<0.02)$, triglycerides $(r=0.29, p<0.004)$, fasting plasma glucose

Table 1. Baseline characteristics of women with a history of gestational diabetes and healthy control subjects

\begin{tabular}{|c|c|c|c|}
\hline & \multicolumn{2}{|c|}{ Gestational diabetic subjects } & \multirow{2}{*}{$\begin{array}{l}\text { Control subjects } \\
(n=17)\end{array}$} \\
\hline & $\left(\mathrm{BMI} \geq 25 \mathrm{~kg} / \mathrm{m}^{2} ; n=46\right)$ & $\left(\mathrm{BMI}<25 \mathrm{~kg} / \mathrm{m}^{2} ; n=31\right)$ & \\
\hline Age (years) & $33 \pm 1$ & $32 \pm 1$ & $30 \pm 1$ \\
\hline Smokers & 7 & 4 & 2 \\
\hline BMI (kg/m2) & $29.5 \pm 0.7 \mathrm{a}, \mathrm{b}$ & $22.9 \pm 0.3^{b}$ & $21.6 \pm 0.5$ \\
\hline MAP (mmHg) & $93 \pm 1^{\mathrm{a}}, \mathrm{b}$ & $87 \pm 1$ & $86 \pm 2$ \\
\hline Triglycerides (mg/dl) & $121 \pm 11^{\mathrm{a}, \mathrm{b}}$ & $79 \pm 6$ & $68 \pm 7$ \\
\hline Cholesterol (mg/dl) & $204 \pm 6$ & $206 \pm 7$ & $207 \pm 16$ \\
\hline HDL cholesterol (mg/dl) & $52 \pm 2 \mathrm{a}, \mathrm{b}$ & $63 \pm 2$ & $66 \pm 3$ \\
\hline LDL cholesterol (mg/dl) & $127 \pm 5$ & $127 \pm 6$ & $128 \pm 14$ \\
\hline Creatinine $(\mathrm{mg} / \mathrm{dl})$ & $0.80 \pm 0.02^{b}$ & $0.83 \pm 0.02^{\mathrm{b}}$ & $0.91 \pm 0.02$ \\
\hline Fasting glucose (mg/dl) & $91 \pm 3^{b}$ & $85 \pm 1$ & $82 \pm 1$ \\
\hline Stimulated glucose (mg/dl) & $159 \pm 7 b$ & $144 \pm 8$ & $104 \pm 5$ \\
\hline Insulin resistance ${ }^{c}$ & $23^{a}, b$ & $9^{b}$ & 0 \\
\hline $\mathrm{ADMA}(\mu \mathrm{mol} / \mathrm{l})$ & $0.58 \pm 0.02^{b}$ & $0.57 \pm 0.02^{b}$ & $0.47 \pm 0.03$ \\
\hline SDMA ( $\mu \mathrm{mol} / 1)$ & $0.51 \pm 0.01^{\mathrm{a}}$ & $0.56 \pm 0.02$ & $0.50 \pm 0.03$ \\
\hline ADMA/SDMA & $1.14 \pm 0.03^{\mathrm{a}, \mathrm{b}}$ & $1.02 \pm 0.03^{\mathrm{b}}$ & $0.95 \pm 0.04$ \\
\hline
\end{tabular}

Data are given as means \pm SEM

${ }^{a} p<0.05$ between GDM groups, $t$ test

${ }^{\mathrm{b}} p<0.05$ vs control subjects, $t$ test c Insulin resistance data were available from 44 and 29 women with $\mathrm{BMI} \geq 25 \mathrm{~kg} / \mathrm{m}^{2}$ and $<25 \mathrm{~kg} / \mathrm{m}^{2}$, respectively 
Table 2. One-year follow up of women with a history of gestational diabetes

\begin{tabular}{lcc}
\hline & \multicolumn{2}{c}{ Gestational diabetes } \\
\cline { 2 - 3 } & $(\mathrm{BMI}$ & $(\mathrm{BMI}$ \\
& $\left.=25 \mathrm{~kg} / \mathrm{m}^{2} ;\right)$ & $<25 \mathrm{~kg} / \mathrm{m}^{2} ;$ \\
& $n=24$ & $n=24)$ \\
\hline Age (years) & $35 \pm 1$ & $33 \pm 1$ \\
Smokers & 5 & 3 \\
BMI $(\mathrm{kg} / \mathrm{m} 2)$ & $29.2 \pm 0.8^{\mathrm{a}}$ & $22.5 \pm 0.4$ \\
MAP (mmHg) & $88 \pm 2$ & $85 \pm 2$ \\
Triglycerides $(\mathrm{mg} / \mathrm{dl})$ & $126 \pm 15$ & $91 \pm 11$ \\
Cholesterol $(\mathrm{mg} / \mathrm{dl})$ & $197 \pm 8$ & $183 \pm 5^{\mathrm{b}}$ \\
HDL cholesterol $(\mathrm{mg} / \mathrm{dl})$ & $48 \pm 3$ & $52 \pm 2^{\mathrm{b}}$ \\
LDL cholesterol $(\mathrm{mg} / \mathrm{dl})$ & $124 \pm 7$ & $112 \pm 5$ \\
Creatinine $(\mathrm{mg} / \mathrm{dl})$ & $0.82 \pm 0.03$ & $0.82 \pm 0.03$ \\
Fasting glucose $(\mathrm{mg} / \mathrm{dl})$ & $94 \pm 3^{\mathrm{a}, \mathrm{b}}$ & $87 \pm 1$ \\
Stimulated glucose $(\mathrm{mg} / \mathrm{dl})$ & $130 \pm 7^{\mathrm{a}}, \mathrm{b}$ & $95 \pm 4^{\mathrm{b}}$ \\
Insulin resistance & $11^{\mathrm{a}}$ & 6 \\
ADMA ( $\mu \mathrm{mol} / \mathrm{l})$ & $0.57 \pm 0.03$ & $0.56 \pm 0.02$ \\
SDMA ( $\mu$ mol/l) & $0.58 \pm 0.04^{\mathrm{b}}$ & $0.58 \pm 0.03$ \\
ADMA/SDMA & $0.99 \pm 0.03^{\mathrm{b}}$ & $0.98 \pm 0.03^{\mathrm{b}}$ \\
\hline
\end{tabular}

Data are given as means \pm SEM

a $p<0.05$ between GDM groups, $t$ test

${ }^{\mathrm{b}} p<0.05$ vs baseline, $t$ test

c Insulin resistance data were available from 17 and 20 women with $\mathrm{BMI} \geq 25 \mathrm{~kg} / \mathrm{m}^{2}$ and $<25 \mathrm{~kg} / \mathrm{m}^{2}$, respectively

$(r=0.21, p<0.05)$ and the stimulated 1-h glucose concentrations $(r=0.29, p<0.005)$ in a pooled analysis. In obese patients, ADMA correlated with triglycerides $(r=0.40, p<0.01)$ and the stimulated 1-h glucose concentrations $(r=0.34, p<0.03)$ SDMA concentrations were overall only slightly correlated with fasting glucose $(r=-0.20, p<0.05)$ but correlated with triglycerides $(r=0.32, p<0.04)$ and HDL $(r=-0.33, p<0.03)$ in obese patients. The ADMA-to-SDMA ratio correlated with BMI $(r=0.49, p<0.001)$, triglycerides $(r=0.20$, $p<0.05)$, HDL cholesterol $(r=-2.7, p<0.007)$, fasting plasma glucose $(r=0.48, p<0.001)$, and stimulated blood glucose concentrations $(r=0.30, p<0.003)$. In the subgroup analysis, the ADMA-to-SDMA ratio correlated with BMI $(r=0.49, p<0.001)$ and the 1 -h stimulated glucose concentration $(r=0.32, p<0.04)$ in obese women. No correlation was observed between any of the other parameters. In a multiple regression analysis containing BMI, triglycerides, fasting glucose and 1-h stimulated blood glucose concentrations, only triglycerides were related to ADMA $(p<0.04)$.

One-year follow up of women with GDM. After 1 year, BMI, fasting and stimulated glucose were higher in obese than in non-adipose women $(p<0.05$, Table 2). More obese GDM patients were insulin resistant than non-adipose patients $(p<0.05)$. ADMA, SDMA and ADMA-to-SDMA ratio were not different between the groups. ADMA concentrations were comparable over time. Within obese GDM patients,
SDMA concentrations increased $(p<0.05)$ and the ADMA-to-SDMA ratio decreased after one year $(p<0.01)$. In the non-adipose GDM group, total cholesterol increased $(p<0.05)$ and HDL, stimulated glucose concentrations, and the ADMA-to-SDMA ratio decreased $(p<0.01)$. Other parameters did not change compared with the baseline (Table 2).

Seven women switched groups from obese to nonadipose and three women changed from the non-adipose to the obese group after 1 year. An additional statistical analysis was done including these patients, but it did not change the significance of the study.

\section{Discussion}

This study is consistent with reports on increased circulating concentrations of ADMA in patients at risk or with vascular dysfunction or arteriosclerosis [7, 10, 13, 25]. Serum concentrations of ADMA were approximately $20 \%$ higher in women with a history of GDM than in healthy women. The increased ADMA concentrations in women with previous GDM shortly after delivery were present when hyperglycaemia was already normalised, and remained persistently increased and higher compared with the age-matched healthy control subjects after 1 year. There is evidence that circulating ADMA concentrations can be increased by accelerated proteolysis of proteins containing methylated arginine residues or by reduced degradation of ADMA by the enzyme dimethylarginine dimethylaminohydrolase (DDAH) [3]. It is not known whether these mechanisms of ADMA increase also apply in GDM.

As expected, insulin resistance was more abundant among obese women. Epidemiological data estimate that the percentage of women with GDM that develop impaired glucose tolerance or Type II diabetes is about $50 \%$ in 5 years [26]. In our cohort, Type II diabetes manifested in only two patients during the 1year follow-up and these women were not included in this study. ADMA concentrations were not related to the insulin sensitivity index or other markers of insulin resistance, while a recent study has reported that insulin resistance, measured with the insulin suppression test (Steady State Plasma Glucose, SSPG) correlated with ADMA concentrations in healthy non-diabetic subjects [19]. This discrepancy could be due to differences between the inhomogenous subject sample studied previously, while our population comprising only women with prior gestational diabetes is more selected. Furthermore, SSPG derives from a complex test. Unlike OGTT and FSIGT that see only glucose administration and endogenous or endo-exogenous insulin action, SSPG involves the use of somatostatin and there is evidence that somatostatin can affect glucose clearance [27]. However, the same weak relation between ADMA and other baseline parameters were 
present in both studies [19]. ADMA concentrations were not associated with metabolic risk factors for insulin resistance, diabetes or arteriosclerosis such as fasting or stimulated glucose or total or fractionated cholesterol, and there was no clear association with BMI or blood pressure. The lack of a correlation with cholesterol has also been reported [18] and there was also no correlation with blood pressure in another study [28]. Associations with systemic blood pressure have been described in other studies [19, 29], but this could be more difficult to detect since increases in total peripheral resistance can be compensated for by changes in cardiac output and larger cohorts could be required to establish a clear relation. However, as these cardiovascular risk factors were in the normal range in most subjects under study, this finding cannot rule out an association of these parameters with ADMA in different patient groups.

It is possible that increased concentrations of ADMA could contribute to the development of impaired endothelial function. So far, no threshold data for ADMA leading to a reduction in endothelial function have been established. In hypercholesterolaemia, an increment of ADMA from $1 \mu \mathrm{mol} / 1$ to $1.5 \mu \mathrm{mol} / \mathrm{l}$ reduced flow-mediated dilatation of the brachial artery from $9 \%$ to $6 \%$ [7]. In patients with Type II diabetes, a high-fat meal resulted in a nearly 2.5 -fold increased mean plasma ADMA concentration and a further deterioration of already impaired endothelial function as estimated by measurement of flow-mediated dilatation of the brachial artery [18]. While we have not assessed vascular function, one would expect a much smaller impairment in our patients accordingly. In a clinical study however, the reduction in flow-mediated brachial artery dilatation was reduced by more than $80 \%$ in women with a history of GDM and was related to insulin resistance [16]. This suggests that ADMA might not be the only contributor to impaired vascular function, but also indicates that even if hyperlipidaemia or hyperglycaemia are absent or appropriately controlled for in women with previous GDM, they could be at risk for endothelial dysfunction on the basis of increased endogenous nitric oxide inhibitors alone.

It is known that insulin resistance is associated with endothelial dysfunction [30], where increased oxidative stress is also detectable [31]. Under normal conditions, the formation of reactive oxygen species is kept under tight control by the capacity of the endogenous antioxidant defence system [32]. The generation of reactive oxygen species can be stimulated by hyperglycaemia and hyperlipidaemia [33] and the production of ADMA in human endothelial cells can also be induced by LDL [34]. The latter effect can be abolished by antioxidant strategies, which supports the concept that oxidative stress could be linked to the development of impaired vascular function in these patients. Incubation of endothelial cells with ADMA also increased formation of superoxide anions [35]. Consistently, short-term oral supplementation with ascorbic acid was able to improve endothelium-dependent vasodilatation in women with GDM [36]. No relation of ADMA concentrations with LDL was seen in our study, but adding other metabolic disorders could cause more incremental damage to endothelial dysfunction induced by ADMA.

SDMA was not consistently increased in women with GDM. However, SDMA at the concentrations observed has no effect on the endothelium [5] and is not associated with cardiovascular risk factors [37]. Unlike ADMA, SDMA is not metabolized by DDAH, and excreted directly into urine [3]. This difference was not found in our study, where serum creatinine concentrations were in the normal range in all groups. This also rules out that renal function could have contributed to the differences seen in dimethylarginine concentrations in our study.

Our findings also suggest that ADMA could represent a sensitive laboratory marker for alterations in the L-arginine-nitric oxide metabolism in GDM patients. A power calculation based on results in the control group estimates that a difference between means as small as $0.1 \mu \mathrm{mol} / 1$ is detectable with $80 \%$ power. It is currently unclear if ADMA could also be used as a surrogate endpoint in clinical trials as suggested from studies in patients with peripheral artery disease receiving intravenous L-arginine therapy [38] or in insulin-resistant patients receiving the insulin sensitizer rosiglitazone [19]. Since specific mimetics or inhibitors of intracellular methyltransferases are also not available at present, it will be difficult to extrapolate clinical findings to ADMA concentrations. The wide range of underlying causes and metabolic derangements that impair vascular function make it unlikely that ADMA targeted therapy alone could reverse abnormal endothelial responsiveness completely. For example, factors such as increased formation of reactive oxygen species also decrease nitric oxide bioavailability and would be unaffected by such drugs.

The methodology of ADMA quantification has not been standardized and concentrations differ slightly between studies. The concentrations seen in our study are in the range of those in patients at risk for acute coronary events [25] or women with pre-eclampsia [12] and their control subjects, respectively. We therefore speculate that increased ADMA in GDM women could indicate a high risk for clinical manifestation of arteriosclerosis in these patients, but studies with hard endpoints are currently not available.

In conclusion, this study shows that serum ADMA concentrations are increased in normoglycaemic women with previous GDM. There is no association of this increase with surrogate markers of insulin resistance or other risk factors for diabetes or cardiovascular events in GDM patients. 
Acknowledgements. This study was supported by a grant from the Fund of the Central Bank of Austria (Jubiläumsfonds der Oesterreichischen Nationalbank) (grant no. 8978, to F. Mittermayer), and from the Austrian Science Fund (grant no. P-14515PAT, to AK-W).

\section{References}

1. Nathan C (1992) Nitric oxide as a secretory product of mammalian cells. FASEB J 6: 3051-3064

2. Moncada S, Higgs A (1993) The L-arginine-nitric oxide pathway. N Engl J Med 329: 2002-2012

3. Leiper J, Vallance P (1999) Biological significance of endogenous methylarginines that inhibit nitric oxide synthases. Cardiovasc Res 43: 542-548

4. Closs EI, Basha FZ, Habermeier A, Forstermann U (1997) Interference of L-arginine analogues with L-arginine transport mediated by the $\mathrm{y}+$ carrier hCAT-2B. Nitric Oxide 1: 65-73

5. Azuma H, Sato J, Hamasaki H, Sugimoto A, Isotani E, Obayashi S (1995) Accumulation of endogenous inhibitors for nitric oxide synthesis and decreased content of L-arginine in regenerated endothelial cells. Br J Pharmacol 115: 1001-1004

6. Bode-Böger SM, Böger RH, Kienke S, Junker W, Frölich JC (1996) Elevated L-arginine/dimethylarginine ratio contributes to enhanced systemic NO production by dietary L-arginine in hypercholesterolemic rabbits. Biochem Biophys Res Commun 219: 598-603

7. Böger RH, Bode-Böger SM, Szuba A et al. (1998) Asymmetric dimethylarginine (ADMA): a novel risk factor for endothelial dysfunction: its role hypercholesterolemia. Circulation 98: 1842-1847

8. Matsuoka H, Itoh S, Kimoto M et al. (1997) Asymmetrical dimethylarginine, an endogenous nitric oxide synthase inhibitor, in experimental hypertension. Hypertension 29: 242-247

9. Surdacki A, Nowicki M, Sandmann J et al. (1999) Reduced urinary excretion of nitric oxide metabolites and increased plasma levels of asymmetric dimethylarginine in men with essential hypertension. J Cardiovasc Pharmacol 33: 652658

10. Böger RH, Bode-Böger SM, Thiele W, Junker W, Alexander K, Frölich JC (1997) Biochemical evidence for impaired nitric oxide synthesis in patients with peripheral arterial occlusive disease. Circulation 95: 20682074

11. Vallance P, Leone A, Calver A, Collier J, Moncada S (1992) Accumulation of an endogenous inhibitor of nitric oxide synthesis in chronic renal failure. Lancet 339: $572-575$

12. Pettersson A, Hedner T, Milsom I (1998) Increased circulating concentrations of asymmetric dimethyl arginine (ADMA), an endogenous inhibitor of nitric oxide synthesis, in preeclampsia. Acta Obstet Gynecol Scand 77: 808-813

13. Walker HA, McGing E, Fisher I et al. (2001) Endotheliumdependent vasodilation is independent of the plasma L-arginine/ADMA ratio in patients with stable angina: lack of effect of oral L-arginine on endothelial function, oxidative stress and exercise performance. J Am Coll Cardiol 38: 499-505

14. Johnstone MT, Creager SJ, Scales KM, Cusco JA, Lee BK, Creager MA (1993) Impaired endothelium-dependent vasodilation in patients with insulin-dependent diabetes mellitus. Circulation 88: 2510-2516
15. McVeigh GE, Brennan GM, Johnston GD et al. (1992) Impaired endothelium-dependent and independent vasodilation in patients with Type II (non-insulin-dependent) diabetes mellitus. Diabetologia 35: 771-776

16. Anastasiou E, Lekakis JP, Alevizaki M et al. (1998) Impaired endothelium-dependent vasodilatation in women with previous gestational diabetes. Diabetes Care 21: 2111-2115

17. Hu J, Norman M, Wallensteen M, Gennser G (1998) Increased large arterial stiffness and impaired acetylcholine induced skin vasodilatation in women with previous gestational diabetes mellitus. $\mathrm{Br} \mathrm{J}$ Obstet Gynaecol 105: 1279-1287

18. Fard A, Tuck CH, Donis JA et al. (2000) Acute elevations of plasma asymmetric dimethylarginine and impaired endothelial function in response to a high-fat meal in patients with type 2 diabetes. Arterioscler Thromb Vasc Biol 20: 2039-2044

19. Stühlinger MC, Abbasi F, Chu JW et al. (2002) Relationship between insulin resistance and an endogenous nitric oxide synthase inhibitor. JAMA 287: 1420-1426

20. Rolandsson O, Hagg E, Nilsson M, Hallmans G, Mincheva-Nilsson L, Lernmark A (2001) Prediction of diabetes with body mass index, oral glucose tolerance test and islet cell autoantibodies in a regional population. J Intern Med 249: 279-288

21. Ferrannini E, Camastra S (1998) Relationship between impaired glucose tolerance, non-insulin-dependent diabetes mellitus and obesity. Eur J Clin Invest 28 [Suppl 2]: 3-7

22. Kuczmarski RJ, Flegal KM (2000) Criteria for definition of overweight in transition: background and recommendations in the United States. Am J Clin Nutr 72: 1074-1081

23. Pacini G, Tonolo G, Sambataro M et al. (1998) Insulin sensitivity and glucose effectiveness: minimal model analysis of regular and insulin-modified FSIGT. Am J Physiol 274: E592-E599

24. Pettersson A, Uggla L, Backman V (1997) Determination of dimethylated arginines in human plasma by high-performance liquid chromatography. J Chromatogr B Biomed Sci Appl 692: 257-262

25. Valkonen VP, Paiva H, Salonen JT et al. (2001) Risk of acute coronary events and serum concentration of asymmetrical dimethylarginine. Lancet 358: 2127-2128

26. Tamas G, Kerenyi Z (2001) Gestational diabetes: current aspects on pathogenesis and treatment. Exp Clin Endocrinol Diabetes 109 [Suppl 2]: S400-5411

27. Bergman RN, Ader M, Finegood DT, Pacini G (1984) Extrapancreatic effect of somatostatin infusion to increase glucose clearance. Am J Physiol 247: E370-E379

28. Anderstam B, Katzarski K, Bergstrom J (1997) Serum levels of NG, NG-dimethyl-L-arginine, a potential endogenous nitric oxide inhibitor in dialysis patients $\mathrm{J}$ Am Soc Nephrol 8: 1437-1442

29. Miyazaki H, Matsuoka H, Cooke JP, Usui M, Ueda S, Okuda S, Imaizumi T (2002) Endogenous nitric oxide synthase inhibitor: a novel marker of atherosclerosis. Circulation 99: 1141-1146

30. Steinberg HO, Chaker H, Leaming R, Johnson A, Brechtel G, Baron AD (1996) Obesity/insulin resistance is associated with endothelial dysfunction. Implications for the syndrome of insulin resistance. J Clin Invest 97: 26012610

31. Ting HH, Timimi FK, Boles KS, Creager SJ, Ganz P, Creager MA (1996) Vitamin C improves endothelium-dependent vasodilation in patients with non-insulin-dependent diabetes mellitus. J Clin Invest 97: 22-28 
32. Cuzzocrea S, Riley DP, Caputi AP, Salvemini D (2001) Antioxidant therapy: a new pharmacological approach in shock, inflammation, and ischemia/reperfusion injury. Pharmacol Rev 53: 135-159

33. Inoguchi T, Li P, Umeda F et al. (2000) High glucose level and free fatty acid stimulate reactive oxygen species production through protein kinase C-dependent activation of $\mathrm{NAD}(\mathrm{P}) \mathrm{H}$ oxidase in cultured vascular cells. Diabetes 49: 1939-1945

34. Böger RH, Sydow K, Borlak J et al. (2000) LDL cholesterol upregulates synthesis of asymmetrical dimethylarginine in human endothelial cells: involvement of S-adenosylmethionine-dependent methyltransferases. Circ Res 87: 99-105

35. Böger RH, Bode-Böger SM, Tsao PS, Lin PS, Chan JR, Cooke JP (2000) An endogenous inhibitor of nitric oxide synthase regulates endothelial adhesiveness for monocytes. J Am Coll Cardiol 36: 2287-2295

36. Lekakis JP, Anastasiou EA, Papamichael CM et al. (2000) Short-term oral ascorbic acid improves endothelium-dependent vasodilatation in women with a history of gestational diabetes mellitus. Diabetes Care 23: 1432-1434

37. Böger RH, Bode-Böger SM (2000) Asymmetric dimethylarginine, derangements of the endothelial nitric oxide synthase pathway, and cardiovascular diseases. Semin Thromb Hemost 26: 539-545

38. Böger RH, Bode-Böger SM, Thiele W, Creutzig A, Alexander K, Frölich JC (1998) Restoring vascular nitric oxide formation by L-arginine improves the symptoms of intermittent claudication in patients with peripheral arterial disease. J Am Coll Cardiol 32: 1336-1344 